\title{
Expression level of microRNA-200c is associated with cell morphology in vitro and histological differentiation through regulation of ZEB1/2 and E-cadherin in gastric carcinoma
}

\author{
ATSUSHI KURATA ${ }^{1}$, MASATOSHI YAMADA ${ }^{1}$, SHIN-ICHIRO OHNO ${ }^{1}$, SHIGERU INOUE ${ }^{2}$, \\ HIROTSUGU HASHIMOTO $^{3}$, KOJI FUJITA ${ }^{1}$, MASAKATSU TAKANASHI ${ }^{1}$ and MASAHIKO KURODA $^{1}$ \\ Departments of ${ }^{1}$ Molecular Pathology and ${ }^{2}$ Preventive Medicine and Public Health, Tokyo Medical University, \\ Tokyo 160-8402; ${ }^{3}$ Department of Diagnostic Pathology, NTT Medical Center Tokyo, Tokyo 141-8625, Japan
}

Received June 27, 2017; Accepted October 30, 2017

DOI: 10.3892/or.2017.6093

\begin{abstract}
Scirrhous type gastric cancer is characterized by diffuse infiltration of poorly differentiated adenocarcinoma cells and poor prognosis. Although association of poorly differentiated histology with reduction in E-cadherin expression, as well as association of microRNA (miR)-200c with E-cadherin through regulation of ZEB1/2, has been reported, participation of miR-200c in gastric carcinogenesis is not fully understood. We used 6 cell lines originating from gastric cancers, and investigated levels of miR-200c along with its target mRNAs ZEB1/2 and E-cadherin by qRT-PCR.ZEB1 and E-cadherin protein expression was also assessed via western blotting. Furthermore, we investigated the expression levels of miR-200c by in situ hybridization, along with the expression of ZEB1 and E-cadherin by immunohistochemistry, in 97 gastric adenocarcinoma tissues. Inverse correlation between miR-200c and ZEB1 levels were obtained by qRT-PCR in cell lines $(\mathrm{P}<0.05)$. Cell lines with low miR-200c and high ZEB1 exhibited low E-cadherin expression in both qRT-PCR and western blotting, and exhibited spindle-shaped morphology, in contrast to round cell morphology in those cell lines with high miR-200c levels. Inverse correlations were also obtained between miR-200c and ZEB1 as well as between ZEB1 and E-cadherin levels in tissue samples $(\mathrm{P}<0.001)$. Cancer tissues with low miR-200c, high ZEB1, and low E-cadherin expression were associated with poorly differentiated histology, in contrast to tubular form in cancers with high miR-200c expression levels $(\mathrm{P}<0.001)$. Our data revealed that downregulation of miR-200c primarily regulated cell morphology by
\end{abstract}

Correspondence to: Dr Atsushi Kurata, Department of Molecular Pathology, Tokyo Medical University, 6-1-1, Shinjuku, Shinjuku-ku, Tokyo 160-8402, Japan

E-mail: akurata@tokyo-med.ac.jp

Key words: gastric adenocarcinoma, microRNA-200c, E-cadherin, epithelial-mesenchymal transition, cell morphology downregulation of E-cadherin through upregulation of ZEB1, leading to poorly differentiated histology in gastric cancer.

\section{Introduction}

Gastric cancer is the fourth most common cancer and the second leading cause of cancer-related deaths in the world (1). More than 980,000 new gastric cancer cases are diagnosed annually, and the disease causes approximately 730,000 deaths per year, with the highest incidence rates in Eastern Asia (1). Scirrhous cancer, which accounts for approximately $10 \%$ of all gastric cancers, is characterized by diffuse infiltration of poorly differentiated adenocarcinoma cells with extensive stromal fibrosis, leading to frequent peritoneal dissemination and extremely poor prognosis $(2,3)$. Molecular analyses have revealed that reduction in the expression of the cell-cell adhesion molecule, E-cadherin, was involved in the development and infiltrative growth of scirrhous type gastric carcinomas $(4,5)$. Reduction in E-cadherin expression observed in poorly differentiated carcinomas of the lungs and endometrium $(6,7)$, promotes detachment of cells from each other and increases cell motility; thus, leading to epithelialmesenchymal transition (EMT) (8).

On the other hand, epigenetic regulation including the role of microRNAs (miRNAs, miRs) in carcinogenesis has recently been intensively studied. miRNAs are endogenous 18-24-nucleotide (nt) single-stranded RNA molecules that act as post-transcriptional regulators of gene expression (9). They stabilize target mRNA transcripts through post-transcriptional gene silencing via either inhibition of the translation process or cleavage of target mRNAs $(10,11)$. miRNAs sharing the same seed sequence are grouped into families and are theorized to target overlapping sets of genes (12). miRNAs play diverse roles in numerous cellular processes; in particular, their expression is altered during tumorigenesis, and they can act as oncogenes or tumor suppressors (13).

Recent studies have shown the importance of certain miRNAs in modulating EMT, and the miR-200 family has been described to be a key regulator of this process $(7,8,14)$. The miR-200 family consists of 5 members: miR-200a, miR-200b and miR-429 which are located on chromosome 
1p36 while miR-200c and miR-429 are located on chromosome 12p13 (14). The miR-200 family suppresses zinc-finger E-box binding homeobox (ZEB) 1 and ZEB2, which in turn inhibit the expression of E-cadherin; thus, the miR-200 family maintains the epithelial phenotype through upregulation of E-cadherin $(7,8,14)$. Among the miR-200 family, loss of miR-200c has been reported to be pivotal to inducing aggressive, invasive and chemoresistant phenotypes in several cancers (15-17). Although downregulation of miR-200a and miR-200b have been reported to be associated with poor prognosis or EBV-association in gastric cancers $(18,19)$, little is known about miR-200c in this context. Recent reports demonstrated that miR-200c may affect E-cadherin expression through regulation of ZEB1/2 expression (20,21); however, the influences of miR-200c on cell morphology and its association with histological type have so far not been reported.

In the present study, we investigated the expression of miR-200c, as well as corresponding targets at both the mRNA and protein levels, in several gastric carcinoma cell lines. In addition, we examined the expression level of miR-200c and respective target proteins in gastric carcinoma tissues and adjacent non-tumor tissues. Our results revealed that the expression level of miR-200c was associated with the morphology of gastric carcinoma cell lines, as well as histological differentiation in cancer tissues, along with the upregulation or downregulation of ZEB $1 / 2$ and E-cadherin.

\section{Materials and methods}

Cell culture and RNA preparation. Six human gastric carcinoma cell lines, H-111-TC, HGC-27, Kato-III, MKN-1, MKN-45 and NU-GC-4 were purchased from Riken Cell Bank (Tsukuba, Japan) and used in the present study. According to the description by the Riken Cell Bank and literature (22-27), these cell lines originated from 1 unknown origin (H-111-TC) and 5 metastatic carcinoma, as well as 2 tubular (well differentiated or adenosquamous) carcinoma (H-111-TC and MKN-1) and 4 poorly differentiated carcinoma including signet ring cell type. HGC-27 and MKN-1 cell lines were maintained in Dulbecco's modified Eagle's medium (DMEM; Gibco-BRL, Rockville, MD, USA), whereas NU-GC-4, Kato-III, MKN-45 and H-111-TC cell lines were maintained in RPMI-1640 (Gibco-BRL), supplemented with $10 \%$ fetal calf serum (FCS), penicillin, and streptomycin, at $37^{\circ} \mathrm{C}$ in a humidified atmosphere of $5 \% \mathrm{CO}_{2}$. When they reached $80-90 \%$ confluence, the cells were washed with phosphate-buffered saline (PBS) and homogenized immediately in Isogen reagent (Nippon Gene, Osaka, Japan). Total RNA was extracted according to the manufacturer's instructions.

Quantitative real-time reverse transcription polymerase chain reaction ( $q R T-P C R$ ) analysis. The RNA samples were suspended in $20 \mu \mathrm{l}$ of nuclease-free water, and miRNA-200c levels were quantified using TaqMan MicroRNA Assay technology (Applied Biosystems; Life Technologies, Carlsbad, CA, USA), as previously described (28). miRNA-200c levels were normalized against levels of RNU6B. The hsa-miR-200c oligonucleotide was a synthetic double-stranded 23-nt RNA, 5'-UAAUACUGCCGGGUAAUGAUGGA-3' and 5'-CGUCU UACCCAGCAGUGUUUGG-3' purchased from BONAC
Corp. (Fukuoka, Japan). To assess the mRNA levels of ZEB1, ZEB2 and E-cadherin, reverse-transcription reactions were performed using M-MLV reverse transcriptase (Invitrogen, Carlsbad, CA, USA). Quantitative PCR (FastStart Universal SYBR-Green Master; Roche, Basel, Switzerland) reactions were run on an Mx3005P thermocycler (Stratagene, La Jolla, CA, USA) and analyzed using MxPro QPCR software, version 4.01 (Stratagene, Agilent Technologies, Santa Clara, CA, USA). The level of gene expression relative to glyceraldehyde 3-phosphate dehydrogenase (GAPDH) was determined. The primer sequences were as follows: ZEB1 forward, 5'-TGTCA CCATGAAACCATTGC-3' and reverse, 5'-AGGTAAAGTG CGCTTCCTCA-3'; ZEB2 forward, 5'-GGGCATTCAGTGA CCTGACA-3' and reverse, 5'-GCATTGTTCCCATAGAGT TC-3'; E-cadherin forward, 5'-TCCTCGGATTCTCTGCT CTC-3' and reverse, 5'-CTCTGACCTTTTGCCAGGAG-3'; GAPDH forward, 5'-ATGGGGAAGGTGAAGGTCG-3' and reverse, 5'-GGGTCATTGATGGCAACAATATC-3'. The experiments were performed 3 times, with the average levels and standard deviations from the mean being obtained. Correlations between average levels of miR-200c, ZEB1, ZEB2 and E-cadherin in all cell lines were estimated by Spearman's rank correlation using the SPSS software package (ver17.0; SPSS Japan Inc., Tokyo, Japan). The results were considered significant if the $\mathrm{P}<0.05$.

Western blot analysis. Cell lines were lysed in RIPA buffer. After boiling with sodium dodecyl sulfate loading buffer, equal amounts $(10 \mu \mathrm{g})$ of the proteins were electrophoresed on $10 \%$ sodium dodecyl sulfate-polyacrylamide gels (SDS-PAGE) and transferred to Immobilon membranes (Millipore Inc., Bedford, MA, USA) by semidry blotting. Five percent milk powder dissolved in PBS buffer containing $0.1 \%$ Tween-20 was used to block non-specific binding. Then, using standard techniques, the membranes were probed with the following antibodies: anti-E-cadherin antibody (mouse monoclonal antibody to human E-cadherin; clone: HECD-1; dilution: 1:200; Takara, Tokyo, Japan), anti-ZEB1 antibody (rabbit monoclonal antibody to ZEB1; clone: D80D3; dilution: 1:100; Cell Signaling Technology, Danvers, MA, USA), and anti- $\beta$-actin antibody (mouse monoclonal antibody to $\beta$-actin; clone: $\mathrm{C} 4$; dilution: 1:1,000; Santa Cruz Biotechnology, Dallas, TX, USA). Antibody-antigen complexes were detected using Immobilon Western chemiluminescent horseradish peroxidase substrate (Millipore), and signals were recorded on a LAS-3000 mini system (Fujifilm, Tokyo, Japan).

Human tissue specimens and tissue microarrays. Human tissue specimens were obtained from 97 patients who underwent gastric cancer surgery at Tokyo Medical University Hospital between 2003 and 2010. The Tokyo Medical University institutional review board approved the present study, and all patients provided written informed consent. The patient cohort included 74 males and 23 females, ranging in age from 40 to 92 years (average 66.3 years). Other clinicopathological characteristics are listed in Table I. The tumors were cut into $\sim 5$-mm sections after fixation in $10 \%$ formalin and embedded in paraffin. From these paraffin blocks, tissue microarrays (TMAs) were constructed, each consisting of 12 specimens of 6-mm diameter on one slide. As previously described (29), 
Table I. Clinical and histopathological features of 97 patients.

\begin{tabular}{lc}
\hline Characteristics & Data \\
\hline Mean age (years) & $66.3 \pm 11.9$ \\
Sex (male/female) & $74 / 23$ \\
Lymph nodes metastasis (+/-) & $33 / 64$ \\
Tumor diameter (mm) & $47.8 \pm 28.2$ \\
Depth (M/SM/MP/SS/SE/SI) & $25 / 26 / 8 / 25 / 9 / 4$ \\
Vessel infiltration (+/-) & $50 / 47$ \\
Histology of tubular formation (+/-) & $67 / 30$
\end{tabular}

M, mucosa; SM, submucosa; MP, muscularis propria; SS, subserosa; SE, serosa exposed; SI, serosa infiltrating (neighboring organ or organs involved).

TMAs allow the relocation of multiple tissue samples from conventional histologic paraffin blocks so that tissues from multiple patients can be analyzed on the same slide.

In situ hybridization. In situ hybridization (ISH) was performed on TMAs containing 3- $\mu \mathrm{m}$ consecutive sections of formalin-fixed, paraffin-embedded (FFPE) samples. Probes utilized were based on 5' digoxigenin (DIG)-labeled ISH locked nucleic acid (LNA) technology (miRCURY-LNA detection probe; Exiqon A/S, Vedbaek, Denmark). Slides were prepared using the Ventana HX System BenchMark (Ventana Medical Systems, Inc., Tucson, AZ, USA). We used the following 5' DIG-labeled LNA probes for ISH: hsa-miR-200c, 5'-TCCATCATTACCCGGCAGTATTA-3'; and non-target negative control sequence, 5'-GTGTAACA CGTCTATACGCCCA-3'.

Immunohistochemistry. Immunohistochemistry (IHC) was performed on TMAs containing 4- $\mu \mathrm{m}$ consecutive sections of FFPE gastric tissue samples. After deparaffinization and rehydration, endogenous peroxidases were blocked by incubation in $0.3 \%$ hydrogen peroxide solution for $20 \mathrm{~min}$. To expose antigens, the sections were autoclaved in EDTA buffer (pH 9.0) for $10 \mathrm{~min}$ and cooled for $30 \mathrm{~min}$. After rinsing in $0.05 \mathrm{M}$ Tris-buffered saline containing $0.1 \%$ Tween-20 $(\mathrm{pH} 7.6)$, the sections were incubated with an affinity-purified anti-ZEB1 antibody (clone: D80D3; dilution: 1:200; Cell Signaling Technology) and anti-E-cadherin antibody (clone: HECD-1; dilution: 1:1,000; Takara) for $3 \mathrm{~h}$ at RT. Samples were then washed three times in PBS and incubated with Dako secondary antibody for $15 \mathrm{~min}$ at RT. Horseradish peroxidase-labeled polymer conjugated to a mixture of goat anti-mouse and anti-rabbit immunoglobulin antibodies (Code: K5007; prediluted; Dako, Glostrup, Denmark) was used as the secondary antibody. 3,3'-Diaminobenzidine tetrachloride (DAB) was used for color development and sections were counterstained with hematoxylin.

Evaluation of tissue images. ISH- or IHC-stained slides were examined by light microscopy using an Olympus BX50 microscope. Three different fields (x200) in each tissue were randomly selected. Digital images of ISH and IHC were captured by the NY-D5000 super system (Microscope Network; Nikon, Tokyo, Japan) and were printed by a truecolor printer (IPSiO SP C420; Ricoh, Tokyo, Japan). Positive ISH results caused the cytoplasm to appear blue, whereas positive IHC results appeared brown. Nuclear immunostaining was assessed for ZEB1, while cytomembranous immunostaining was assessed for E-cadherin. Images were interpreted semiquantitatively by assessing the extent and intensity of staining on the entirety of each tissue section present on the slides, as previously described (30). Briefly, the total percentage of positively-stained tumor cells was first determined. Then, the percentage of weakly-, moderately- and strongly-stained cells was determined, so that the sum of these categories equated with the overall percentage of positivity. A final staining score was then calculated as the sum of $1 \mathrm{x}$ percentage of weak, $2 \mathrm{x}$ percentage of moderate, and $3 \times$ percentage of strong staining (maximum score $=300$ ).

Statistical analyses of data from human tissue studies. Prior to statistical analyses, clinicopathlogical data as well as tissue image results were dichotomized into two groups of 'high' and 'low' levels, according to the median values. Thus, the final score for staining of miR-200c was used to define high-level expression (final score $\geq 201$ ) and low-level expression (final score $<201$ ). Likewise, the final score for staining of ZEB1 was used to define high-level expression (final score $\geq 1$ ) and low-level expression (final score $<1$ ), as well as that of E-cadherin used to define high-level expression (final score $\geq 111$ ) and low-level expression (final score $<111)$. Tumor diameter was dichotomized as either highlevel (diameter $\geq 40 \mathrm{~mm}$ ) or low-level (diameter $<40 \mathrm{~mm}$ ). High-age ( $\geq 65$ years old) and low-age ( $<65$ years old) groups were established according to convention, as were advanced (depth $\geq \mathrm{mp}$ ) and early (depth $<\mathrm{mp}$ ) cancer groups. Histologically, both a well/moderately differentiated tubular adenocarcinoma group and a poorly differentiated adenocarcinoma group were established. Then, statistical analyses including Chi-square test and multivariate analysis using logistic regression models were performed using the SPSS software package (ver17.0; SPSS). Results were considered significant if the $\mathrm{P}<0.05$.

\section{Results}

Quantitative analysis of miR-200c and target $m R N A$ expression in gastric carcinoma cell lines. We investigated miR-200c expression in 6 gastric carcinoma cell lines by qRT-PCR analysis. As shown in Fig. 1A, the expression level of miR-200c descended in the following order (from high to low): Kato-III > MKN-45 > NU-GC-4 > H-111-TC > HGC-27 $>$ MKN-1. Of note, the latter 2 cell lines HGC-27 and MKN-1 expressed very low levels of miR-200c. We also investigated the expression of particular target mRNAs, namely ZEB1 and ZEB2, along with E-cadherin, in the same cell lines. The expression level of ZEB1 descended in the following order (from high to low): HGC-27 > MKN-1 > NU-GC-4 > H-111-TC $>$ MKN-45 > Kato-III (Fig. 1B). Of note, the 2 cell lines in which miR-200c expression level was very low exhibited the greatest expression level of ZEB1. Similarly, the expression level of ZEB2 descended in the following order (from high to 


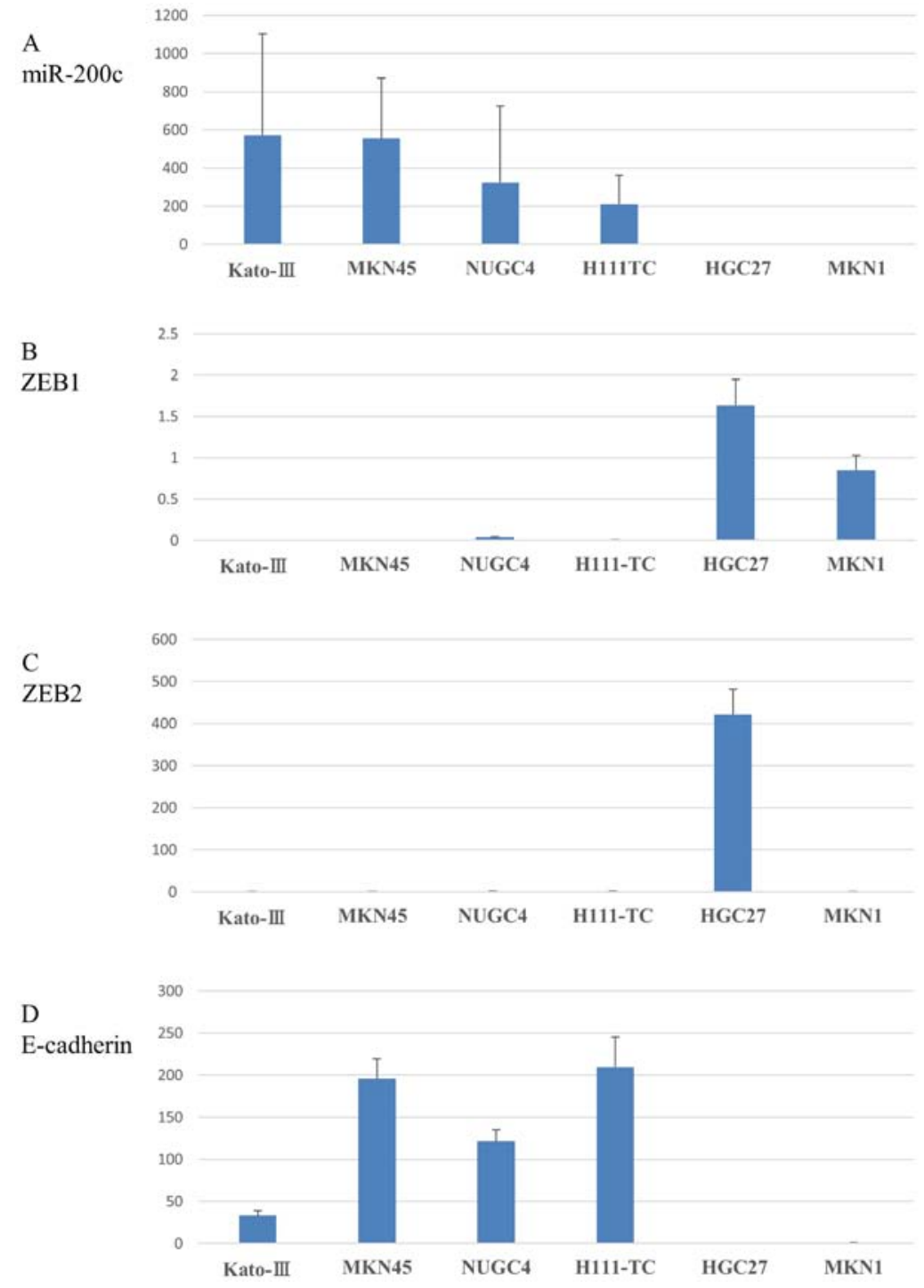

Figure 1. The level of miR-200c and respective target mRNAs in gastric carcinoma cell lines. (A) Gastric carcinoma cell lines were arranged according to the expression level of miR-200c. The expression levels of (B) ZEB1, (C) ZEB2 and (D) E-cadherin expression in gastric carcinoma cell lines arranged according to the level of miR-200c expression.

low): HGC-27 > H-111-TC > NU-GC-4 > MKN-45 > MKN-1 $>$ Kato-III (Fig. 1C). Here, one of the 2 cell lines in which miR-200c expression level was very low exhibited the greatest expression level of ZEB2. On the other hand, the expression level of E-cadherin descended in the following order (from high to low): H-111-TC > MKN-45 > NU-GC-4 > Kato-III > MKN-1 > HGC-27 (Fig. 1D). Of note, the 2 cell lines in which the expression level of miR-200c was very low exhibited the lowest expression level of E-cadherin. As shown in Table II, an inverse correlation was observed between miR-200c and ZEB1 ( $\mathrm{P}<0.05$; by Spearman's rank correlation).

ZEB1 and E-cadherin protein expression in gastric carcinoma cell lines. The expression of ZEB1 and E-cadherin proteins was examined in the 6 human gastric carcinoma cell lines mentioned thus far by western blot analysis. As shown in Fig. 2, expression of ZEB1 was only detected in HGC-27 and MKN-1 cells, which exhibited the lowest expression level of miR-200c determined by qRT-PCR analysis. Conversely, the expression of E-cadherin was not detected in these 2 cell lines;

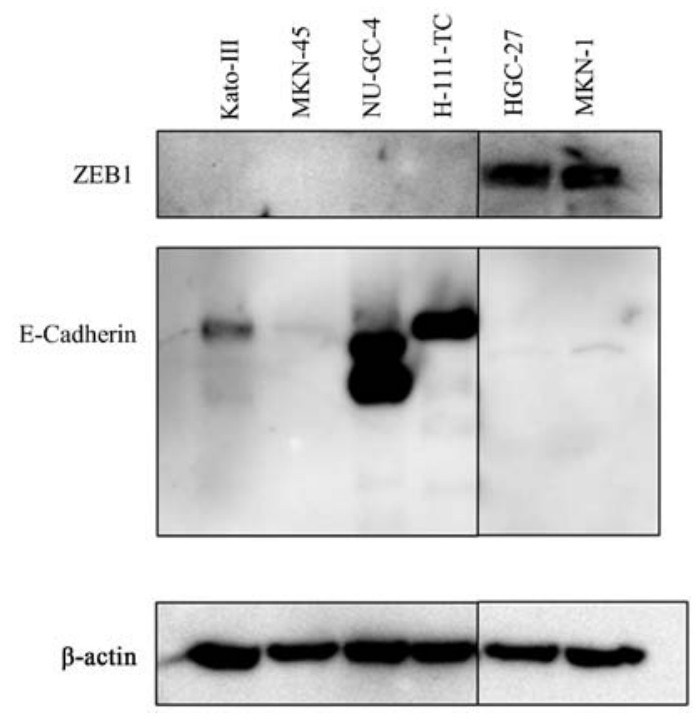

Figure 2. Expression of ZEB1, E-cadherin and $\beta$-actin proteins in gastric carcinoma cell lines, presented by western blot analysis performed on $10 \mu \mathrm{g}$ of the cell extract. 
Table II. Correlations between the average expression levels of miR-200c, ZEB1, ZEB2 and E-cadherin in all cell lines estimated by Spearman's rank correlation.

\begin{tabular}{|c|c|c|c|c|c|c|}
\hline & \multicolumn{2}{|l|}{ ZEB1 } & \multicolumn{2}{|l|}{ ZEB2 } & \multicolumn{2}{|l|}{ E-cadherin } \\
\hline & Correlation coefficient & P-value & Correlation coefficient & $\mathrm{P}$-value & Correlation coefficient & P-value \\
\hline miR-200c & -0.886 & 0.019 & -0.429 & 0.397 & 0.429 & 0.397 \\
\hline ZEB1 & & & 0.600 & 0.208 & -0.600 & 0.208 \\
\hline ZEB2 & & & & & 0.029 & 0.957 \\
\hline
\end{tabular}

Bold indicates $\mathrm{P}<0.05$.

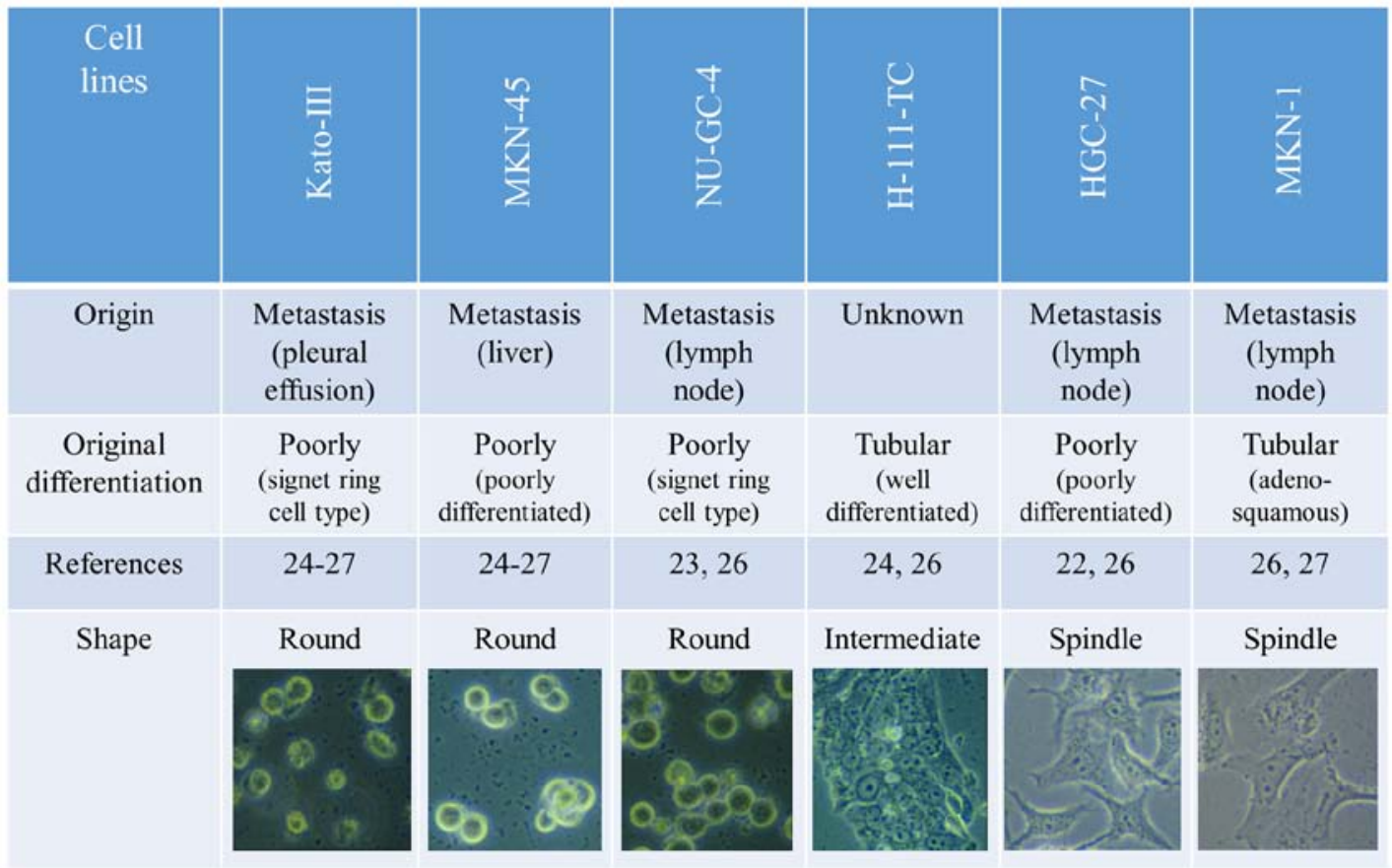

Figure 3. Six gastric carcinoma cell lines arranged according to expression the level of miR-200c. This order is compared with the origin (primary cancer or metastatic cancer), differentiation state (tubular or poorly differentiated), and shape (round/intermediate/spindle) of the cell lines.

instead, E-cadherin expression was detected in NU-GC-4 and $\mathrm{H}-111-\mathrm{TC}$ cell lines, although very weak expression was also detected in Kato-III cells.

Interpretation of divergent expression levels of miR-200c, $Z E B 1 / 2$, and E-cadherin in different gastric carcinoma cell lines. The results of qRT-PCR analyses and western blot analysis were in agreement. Cell lines with a high expression level of miR-200c exhibited low ZEB1/2 and high E-cadherin expression, whereas those with a low expression level of miR-200c exhibited high ZEB1/2 and low E-cadherin expression. Therefore, we investigated characteristics that illustrated differences between the group of cell lines with high miR-200c expression and those with low miR-200c expression. As shown in Fig. 3, we first compared the tumor origin (primary or metastatic tumor) of the cell lines. However, the tumor origin did not clearly correspond to the levels of miR-200c expression, as cell lines derived from metastatic tumors exhibited both low and high miR-200c expression levels. Secondly, we compared the histological differentiation state of the tumors from which the cell lines were derived was compared. Although cell lines from poorly differentiated tumors exhibited high miR-200c expression, the results were inconclusive as one of the cell lines with low miR-200c expression (HGC-27) was originally from a poorly differentiated tumor. Thirdly, we revealed that the morphology of the cell lines was well correlated with the expression level of miR-200c. Cell lines with high miR-200c expression (Kato-III, MKN-45 and NU-GC-4) were roundshaped, those with low miR-200c expression (HGC-27 and MKN-1) were spindle-shaped, and those with intermediate miR-200c expression (H-111-TC) exhibited a phenotype which was a mid-way between round- and spindle-shaped (polygonal or short spindle-shaped).

miR-200c expression patterns in gastric adenocarcinoma and adjacent non-tumor tissues. In human tissue samples, positive miR-200c expression, as determined by ISH, was denoted by cytoplasmic granular blue staining. Most of the normal tubules 


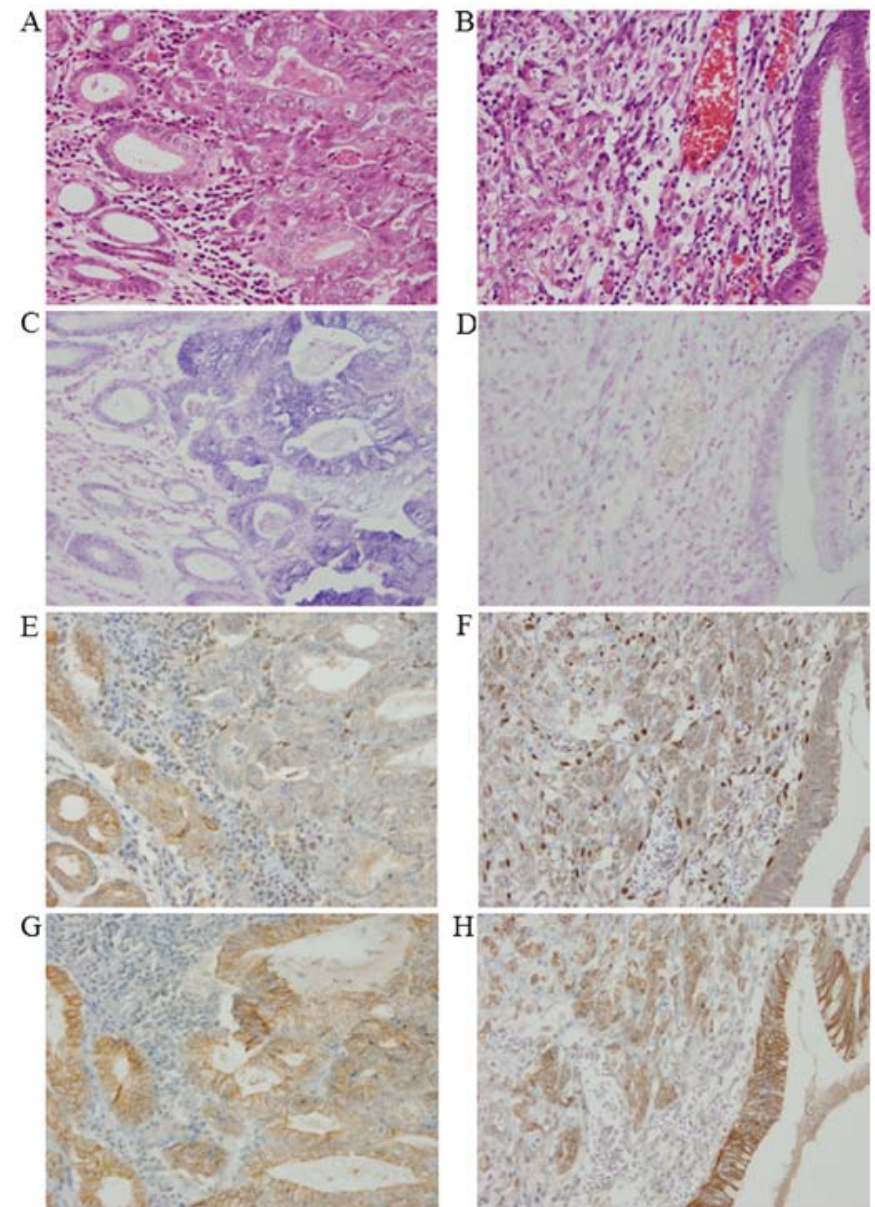

Figure 4. Representative photomicrographs including in situ hybridization and immunohistochemistry. (A and B) Staining with $\mathrm{H} \& \mathrm{E}$ and staining for $(\mathrm{C}$ and D) miR-200c, (E and F) ZEB1 and (G and H) E-cadherin in (A, C, E and G) tubular adenocarcinoma and (B, D, F and $\mathrm{H})$ poorly differentiated adenocarcinoma. Original magnification, x200. (A) Left side are normal glands, right side is carcinoma. (B) Left side is carcinoma, right side is normal foveolar epithelium. (C) Cytoplasmic granular staining in tubular adenocarcinoma was stronger than that found in normal glands. (D) Cytoplasmic granular staining in poorly differentiated adenocarcinoma was weaker than that observed in normal epithelium. (E) Nuclear immunostaining was negative in tubular adenocarcinoma. (F) Nuclear immunostaining was weak but positive in poorly differentiated adenocarcinoma. (G) Cytomembranous immunostaining in tubular adenocarcinoma was as strong as that found in normal glands. (H) Cytomembranous immunostaining in poorly differentiated adenocarcinoma was weaker than that observed in normal epithelium.

stained positive for miR-200c, whereas stromal tissue was not stained. As shown in Fig. 4, miR-200c expression patterns in gastric adenocarcinoma differed substantially between tubular adenocarcinoma and poorly differentiated adenocarcinoma. In tubular adenocarcinoma, miR-200c expression was observed with an intensity level either stronger than or the same as normal tubules. Conversely, in poorly differentiated adenocarcinoma, miR-200c expression was either not identified or observed with weaker staining patterns than normal tubules. The average staining score for miR-200c was $265 \pm 81.6$ in tubular adenocarcinomas, while it was $104 \pm 75.2$ in poorly differentiated adenocarcinomas.

ZEB1 and E-cadherin expression patterns in gastric adenocarcinoma and adjacent non-tumor tissues. Positive ZEB1

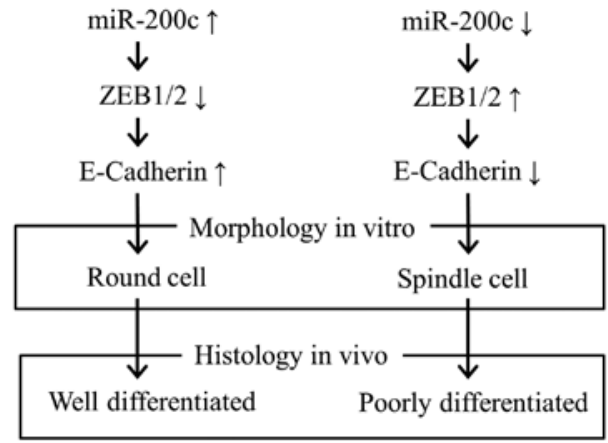

Figure 5. Upregulation/downregulation of miR-200c affects cell morphology and histology.

protein expression was denoted by nuclear staining. Overall positivity of ZEB1 was relatively low in normal tubules and gastric carcinomas. ZEB1 was almost negative in tubular adenocarcinomas; however, positive expression of ZEB1, although generally weak, was sporadically observed in poorly differentiated adenocarcinomas. The average staining score for ZEB1 was $0.84 \pm 2.93$ in tubular adenocarcinomas, while it was $5.8 \pm 8.6$ in poorly differentiated adenocarcinomas. Positive E-cadherin protein expression was indicated by cytomembranous staining. Normal tubules and tubular adenocarcinomas were strongly stained, while poorly differentiated adenocarcinomas were weakly stained (Fig. 4). The average staining score for E-cadherin was $146 \pm 52$ in tubular adenocarcinomas, while it was $39 \pm 38.6$ in poorly differentiated adenocarcinomas.

Interpretation of statistical analyses of human tissue studies. Use of the Chi-square test revealed a correlation between miR-200c and E-cadherin expression levels $(\mathrm{P}<0.001)$ and an inverse correlation between miR-200c and ZEB1 expression levels $(\mathrm{P}<0.001)$ as well as between $\mathrm{ZEB} 1$ and E-cadherin expression levels $(\mathrm{P}<0.001)$. We further compared the expression levels of miR-200c, ZEB1 and E-cadherin with clinicopathological factors (Table III). High expression levels of miR-200c were correlated with older age ( $\geq 65$ years old) $(\mathrm{P}=0.002)$ and tubular histology $(\mathrm{P}<0.001)$. High expression levels of ZEB1 were correlated with poorly differentiated histology $(\mathrm{P}<0.001)$ and advanced stage $(\mathrm{P}=0.040)$. High expression levels of E-cadherin were correlated with older age ( $\geq 65$ years old) $(\mathrm{P}=0.039)$ and tubular histology $(\mathrm{P}<0.001)$. In the multivariate analysis (Table IV), among the clinicopathological factors significantly correlated with the expression levels of miR-200c, ZEB1 and E-cadherin, there was a significant association between the expression level of ZEB1 and advanced stage $(\mathrm{P}=0.031)$, as well as a significant correlation between the expression level of ZEB1 and histology $(\mathrm{P}<0.001)$.

\section{Discussion}

In the present study, we revealed inverse correlations between miR-200c and ZEB1 in both cell lines and tissues, as well as inverse correlations between ZEB1 and E-cadherin in tissues, in gastric carcinoma. These well known correlations which are associated with EMT (8) have been demonstrated both in vitro and in vivo in several cancers including non-small cell lung cancer and pancreatic cancer $(15,16)$ and recently also in 
Table III. Correlations of the expression levels of miR-200c, ZEB1 and E-cadherin with the clinicopathological factors as determined by Chi-squared test.

\begin{tabular}{|c|c|c|c|c|c|c|c|c|c|}
\hline & \multicolumn{3}{|c|}{ miR-200c } & \multicolumn{3}{|c|}{ ZEB1 } & \multicolumn{3}{|c|}{ E-cadherin } \\
\hline & High & Low & P-value & High & Low & P-value & High & Low & P-value \\
\hline Sex & & & 0.474 & & & 0.419 & & & 0.812 \\
\hline Male & 37 & 37 & & 18 & 56 & & 35 & 39 & \\
\hline Female & 9 & 14 & & 8 & 15 & & 12 & 11 & \\
\hline Age (years old) & & & 0.002 & & & 0.063 & & & 0.039 \\
\hline$\geq 65$ & 35 & 22 & & 11 & 46 & & 33 & 24 & \\
\hline$<65$ & 11 & 29 & & 15 & 25 & & 14 & 26 & \\
\hline LN metastasis & & & 0.289 & & & 1.000 & & & 0.830 \\
\hline Positive & 13 & 20 & & 9 & 24 & & 15 & 18 & \\
\hline Negative & 33 & 31 & & 17 & 47 & & 32 & 32 & \\
\hline Histology & & & $<0.001$ & & & $<0.001$ & & & $<0.001$ \\
\hline Tubular & 45 & 22 & & 9 & 58 & & 45 & 22 & \\
\hline Poor differentiation & 1 & 29 & & 17 & 13 & & 2 & 28 & \\
\hline Tumor size (mm) & & & 0.224 & & & 0.360 & & & 0.312 \\
\hline$\geq 40$ & 19 & 28 & & 15 & 32 & & 20 & 27 & \\
\hline$<40$ & 27 & 23 & & 11 & 39 & & 27 & 23 & \\
\hline Stage & & & 0.310 & & & 0.040 & & & 0.840 \\
\hline Advanced & 19 & 27 & & 17 & 29 & & 23 & 23 & \\
\hline Early & 27 & 24 & & 9 & 42 & & 24 & 2 & \\
\hline Vessel invasion & & & 0.157 & & & 0.259 & & & 0.840 \\
\hline Positive & 20 & 30 & & 16 & 34 & & 25 & 25 & \\
\hline Negative & 26 & 21 & & 10 & 37 & & 22 & 25 & \\
\hline
\end{tabular}

LN, lymph node. Bold indicates $\mathrm{P}<0.05$.

Table IV. Multivariate analyses of correlations between the clinicopathological factors and positive expression of miR-200c, ZEB1 and E-cadherin.

\begin{tabular}{|c|c|c|c|c|c|c|}
\hline \multirow[b]{2}{*}{ Clinicopathological factors } & \multicolumn{2}{|c|}{ miR-200c } & \multicolumn{2}{|c|}{ ZEB1 } & \multicolumn{2}{|c|}{ E-cadherin } \\
\hline & OR $(95 \% \mathrm{CI})$ & P-value & OR $(95 \%$ CI $)$ & P-value & OR $(95 \% \mathrm{CI})$ & P-value \\
\hline $\begin{array}{l}\text { Sex } \\
\text { Male vs. female }\end{array}$ & $\begin{array}{c}1.822 \\
(0.505-6.568)\end{array}$ & 0.359 & $\begin{array}{c}0.294 \\
(0.074-1.170)\end{array}$ & 0.082 & $\begin{array}{c}0.438 \\
(0.124-1.553)\end{array}$ & 0.201 \\
\hline $\begin{array}{l}\text { Age }(\text { years old }) \\
\geq 65 \text { vs. }<65\end{array}$ & $\begin{array}{c}2.742 \\
(0.916-8.202)\end{array}$ & 0.071 & $\begin{array}{c}0.505 \\
(0.153-1.662)\end{array}$ & 0.261 & $\begin{array}{c}1.085 \\
(0.370-3.184)\end{array}$ & 0.882 \\
\hline $\begin{array}{l}\text { LN metastasis } \\
\text { Positive vs. negative }\end{array}$ & $\begin{array}{c}1.038 \\
(0.249-4.320)\end{array}$ & 0.959 & $\begin{array}{c}0.347 \\
(0.090-1.342)\end{array}$ & 0.125 & $\begin{array}{c}0.458 \\
(0.111-1.888)\end{array}$ & 0.280 \\
\hline $\begin{array}{l}\text { Histology } \\
\text { Tubular vs. poor differentiation }\end{array}$ & $\begin{array}{c}0.023 \\
(0.003-0.182)\end{array}$ & $<0.001$ & $\begin{array}{c}7.556 \\
(2.471-23.109)\end{array}$ & $<0.001$ & $\begin{array}{c}0.025 \\
(0.005-0.137)\end{array}$ & $<0.001$ \\
\hline $\begin{array}{l}\text { Tumor size }(\mathrm{mm}) \\
\geq 40 \text { vs. }<40\end{array}$ & $\begin{array}{c}1.087 \\
(0.337-3.509)\end{array}$ & 0.889 & $\begin{array}{c}0.809 \\
(0.242-2.703)\end{array}$ & 0.730 & $\begin{array}{c}0.533 \\
(0.172-1.652)\end{array}$ & 0.276 \\
\hline $\begin{array}{l}\text { Stage } \\
\text { Advanced vs. early }\end{array}$ & $\begin{array}{c}1.073 \\
(0.258-4.473)\end{array}$ & 0.923 & $\begin{array}{c}5.522 \\
(1.166-26.148)\end{array}$ & $\mathbf{0 . 0 3 1}$ & $\begin{array}{c}2.197 \\
(0.558-8.653)\end{array}$ & 0.260 \\
\hline $\begin{array}{l}\text { Vessel invasion } \\
\text { Positive vs. negative }\end{array}$ & $\begin{array}{c}0.484 \\
(0.105-2.240)\end{array}$ & 0.354 & $\begin{array}{c}0.998 \\
(0.227-4.378)\end{array}$ & 0.998 & $\begin{array}{c}2.182 \\
(0.489-9.732)\end{array}$ & 0.306 \\
\hline
\end{tabular}

LN, lymph node; OR, odds ratio; CI, confidence interval. Bold indicates $\mathrm{P}<0.05$. 
gastric cancer $(21,31)$. However, this study is the first to have demonstrated that this phenomenon was associated with the morphology of cell lines and histological differentiation, the latter as visualized by ISH, in gastric carcinoma.

qRT-PCR analysis of 6 cell lines in the present study revealed that the expression level of miR-200c descended in the following order: Kato-III $>$ MKN-45 > NU-GC-4 $>\mathrm{H}-111-\mathrm{TC}>\mathrm{HGC}-27>\mathrm{MKN}-1$. The expression level of ZEB1 or ZEB2 corresponded to the reverse order, and that of E-cadherin corresponded to this order. Indeed, significantly inverse correlations between miR-200c and ZEB1 were obtained by Spearman's rank correlation. Our results are in line with a previous study in which E-cadherin gene expression was present in MKN-45 cells but absent in HGC-27 cells (32), along with a recent study which revealed that Kato-III, MKN-45 and NU-GC-4 cells had high expression of E-cadherin and low expression of ZEB1, while MKN-1 cells had low expression of E-cadherin and high expression of ZEB1 (33). However, these authors did not mention any differences in cell morphology in their studies. In the present study, western blot analysis coincided with qRT-PCR data, albeit E-cadherin expression in Kato-III and MKN-45 cells was almost absent contrary to positive expression as determined by qRT-PCR analysis. This result was compatible with another study that revealed that E-cadherin protein expression was undetectable by western blot analysis, while it was detected at the DNA and mRNA level by southern and northern blot analysis, respectively, in Kato-III and MKN-45 cells (4). They mentioned that the possibility of post-translational modification or minor abnormalities in antibody-recognition sites may be involved in this discrepancy.

We searched for key differences in the characteristics between cell lines displaying divergent expression levels of miR-200c which also corresponded to differences in the expression levels of ZEB1/2 and E-cadherin. Although histological differences were conspicuous in the tissue studies mentioned below, cell line groupings based on the expression of miR-200c and associated targets did not correspond to histological differentiation of the derived tumors. Other authors have also demonstrated that E-cadherin was 'unexpectedly' detected in cell lines that originated from poorly differentiated gastric carcinomas (26). This may be due to the fact that cell lines have generally been established from tumors exhibiting heterogenous histology. Indeed, it has been reported that histological difference in original tumors did not influence the growth rate of various cell lines in gastric carcinomas (25). Instead, we established that cell lines with high miR-200c expression (Kato-III, MKN-45 and NU-GC-4), low miR-200c expression (HGC-27 and MKN-1), and intermediate miR-200c expression (H-111-TC) exhibited round, spindle, and intermediate, respectively, cell morphologies. Although studies concerning cell morphology in association with expression of miR-200 family members are scant, one research group demonstrated that expression of miR-200c was associated with round cell morphology whereas that of miR-200a was associated with spindle cell morphology in melanoma cell lines (34). The phenomenon where E-cadherin $(+)$ cells are round and E-cadherin (-) cells are spindle in shape has been reported in esophageal cancer cells and, recently, in colon cancer cells $(35,36)$. Furthermore, it was formerly reported that cell morphology of E-cadherin-deficient cell lines was generally altered from the fibroblastic cell type to the epithelial cell type by transfection with complementary DNA that codes for E-cadherin (37). Therefore, the influence of miR-200c and E-cadherin on cell morphology may be a universal phenomenon.

In the tissue samples, miR-200c expression, as visualized by ISH, was strongly expressed in tubular adenocarcinomas but weakly expressed in poorly differentiated adenocarcinomas. This result was in agreement with a recent study in which low expression levels of each member of the miR-200 family were associated with histological grade in gastric carcinoma tissues, as assessed by qRT-PCR analysis (31). Analysis of non-small cell lung cancer tissue also revealed that low miR-200c expression levels were associated with poorly differentiated histology (15). Assessment of endometrial carcinosarcoma tissues revealed that members of the miR-200 family were downregulated in the mesenchymal part of the tumor (38). Therefore, it is likely that the expression of miR-200c is associated with epithelial histology of tumors in general.

IHC analysis revealed that tubular adenocarcinomas were negative for ZEB1 and positive for E-cadherin, while poorly differentiated adenocarcinomas were sporadically positive for ZEB1 and negative for E-cadherin. Notably, it has long been pointed out that the level of E-cadherin is significantly lower in poorly differentiated adenocarcinomas compared to differentiated adnocarcinomas and non-neoplastic mucosa of the stomach $(4,32)$. The reason for this phenomenon was proposed to be inactivation of the E-cadherin gene due to either a classic two-hit mechanism or hypermethylation (32). In the present study, we add EMT mediated by miR-200c as a possible causal mechanism, since miR-200c and ZEB1 expression levels, as well as ZEB1 and E-cadherin expression levels, were inversely correlated $(\mathrm{P}<0.001)$. Other authors have also demonstrated an inverse correlation between ZEB1 and E-cadherin mRNA expression in gastric cancer tissue (33). Association of poorly differentiated histology with high expression of ZEB1 and low expression of E-cadherin, as determined by IHC, has also been demonstrated in endometrioid carcinoma and non-small cell lung cancers $(6,7)$.

In addition to histology, older age was also correlated with high expression levels of miR-200c and E-cadherin as assessed by Chi-squared test. However, in terms of multivariate analyses, age was not correlated with miR-200c and E-cadherin expression, although histology remained correlated. These results coincide with a well-known fact that poorly differentiated scirrhous gastric carcinoma is more frequent in younger patients $(2,3)$. Conversely, a high expression level of ZEB1 was correlated with advanced stage by multivariate analyses. As other authors have also reported, gastric cancer patients with high ZEB1 mRNA expression displayed significantly poorer survival than those with low expression (33), ZEB1 may be independently associated with advanced stage.

Concerning miR-200c expression in tumors in general, a meta-analysis revealed that low expression of miR-200c in primary cancer tissue from early-stage patients was significantly associated with poor survival (39). Association of low miR-200c expression with high propensity to metastasis, along with highly invasive/aggressive ability, has been demonstrated in several cancers including non-small cell lung cancer and 
pancreatic cancer $(15,16)$. Recent studies have also demonstrated that migration/invasion abilities of gastric cancer cells were promoted by decreased expression of miR-200c, while they were inhibited by ZEB1 siRNA $(21,33)$. In addition, the present study demonstrated an association of miR-200c with cell morphology and histological differentiation of the tumor. Since it has been proposed that the ability of miR-200c to regulate morphological plasticity may be important in relation to dissemination capacity in melanoma (34), we postulate that downregulation of miR-200c primarily regulates cell morphology by downregulation of E-cadherin through upregulation of ZEB1/2, with this morphological change causing poorly differentiated histology (Fig. 5), producing enhanced migration/invasion abilities, and finally leading to scirrhous stromal reaction and poor prognosis $(2,3)$.

In summary, using cell culture models and histological assessment of gastric carcinoma, we demonstrated an inverse correlation in the expression level between miR-200c and ZEB1 in vitro and in vivo, as well as between $\mathrm{ZEB} 1$ and E-cadherin in vivo. Low miR-200c expression was associated with spindle cell morphology in vitro and poorly differentiated histology in vivo; thus, this factor may be involved in the carcinogenesis of scirrhous gastric carcinoma.

\section{Acknowledgements}

The present study was supported by Grants-in-Aid for scientific research (C) (grant no. 24590431).

\section{References}

1. Jemal A, Bray F, Center MM, Ferlay J, Ward E and Forman D: Global cancer statistics. CA Cancer J Clin 61: 69-90, 2011.

2. Duarte I and Llanos O: Patterns of metastases in intestinal and diffuse types of carcinoma of the stomach. Hum Pathol 12: 237-242, 1981.

3. Yokota T, Teshima S, Saito T, Kikuchi S, Kunii Y and Yamauchi H: Borrmann's type IV gastric cancer: Clinicopathologic analysis. Can J Surg 42: 371-376, 1999.

4. Yasui W, Kuniyasu H, Akama Y, Kitahara K, Nagafuchi A, Ishihara S, Tsukita $\mathrm{S}$ and Tahara E: Expression of e-cadherin, alpha-catenins and Beta-catenins in human gastric carcinomas - correlation with histology and tumor progression. Oncol Rep 2: 111-117, 1995.

5. Hippo Y, Yashiro M, Ishii M, Taniguchi H, Tsutsumi S, Hirakawa K, Kodama T and Aburatani H: Differential gene expression profiles of scirrhous gastric cancer cells with high metastatic potential to peritoneum or lymph nodes. Cancer Res 61: 889-895, 2001

6. Matsubara D, Kishaba Y, Yoshimoto T, Sakuma Y, Sakatani T, Tamura T, Endo S, Sugiyama Y, Murakami Y and Niki T: Immunohistochemical analysis of the expression of E-cadherin and ZEB1 in non-small cell lung cancer. Pathol Int 64: 560-568, 2014.

7. Romero-Pérez L, López-García MÁ, Díaz-Martín J, Biscuola M, Castilla MÁ, Tafe LJ, Garg K, Oliva E, Matias-Guiu X, Soslow RA, et al: ZEB1 overexpression associated with E-cadherin and microRNA-200 downregulation is characteristic of undifferentiated endometrial carcinoma. Mod Pathol 26: 1514-1524, 2013

8. Burk U, Schubert J, Wellner U, Schmalhofer O, Vincan E, Spaderna $S$ and Brabletz T: A reciprocal repression between ZEB1 and members of the miR-200 family promotes EMT and invasion in cancer cells. EMBO Rep 9: 582-589, 2008.

9. Poliseno L, Salmena L, Riccardi L, Fornari A, Song MS, Hobbs RM, Sportoletti P, Varmeh S, Egia A, Fedele G, et al: Identification of the miR-106b 25 microRNA cluster as a protooncogenic PTEN-targeting intron that cooperates with its host gene MCM7 in transformation. Sci Signal 3: ra29, 2010.
10. Sethi S, Kong D, Land S, Dyson G, Sakr WA and Sarkar FH: Comprehensive molecular oncogenomic profiling and miRNA analysis of prostate cancer. Am J Transl Res 5: 200-211, 2013.

11. Hassan O, Ahmad A, Sethi S and Sarkar FH: Recent updates on the role of microRNAs in prostate cancer. J Hematol Oncol 5: 9, 2012.

12. Landgraf P, Rusu M, Sheridan R, Sewer A, Iovino N, Aravin A, Pfeffer S, Rice A, Kamphorst AO, Landthaler M, et al: A mammalian microRNA expression atlas based on small RNA library sequencing. Cell 129: 1401-1414, 2007.

13. Ventura A and Jacks T: MicroRNAs and cancer: Short RNAs go a long way. Cell 136: 586-591, 2009.

14. Bojmar L, Karlsson E, Ellegård S, Olsson H, Björnsson B, Hallböök O, Larsson M, Stål O and Sandström P: The role of microRNA-200 in progression of human colorectal and breast cancer. PLoS One 8: e84815, 2013.

15. Ceppi P, Mudduluru G, Kumarswamy R, Rapa I, Scagliotti GV, Papotti $\mathrm{M}$ and Allgayer $\mathrm{H}$ : Loss of miR-200c expression induces an aggressive, invasive, and chemoresistant phenotype in non-small cell lung cancer. Mol Cancer Res 8: 1207-1216, 2010.

16. Yu J, Ohuchida K, Mizumoto K, Sato N, Kayashima T, Fujita H, Nakata K and Tanaka M: MicroRNA, hsa-miR-200c, is an independent prognostic factor in pancreatic cancer and its upregulation inhibits pancreatic cancer invasion but increases cell proliferation. Mol Cancer 9: 169, 2010.

17. Marchini S, Cavalieri D, Fruscio R, Calura E, Garavaglia D, Fuso Nerini I, Mangioni C, Cattoretti G, Clivio L, Beltrame L, et al: Association between miR-200c and the survival of patients with stage I epithelial ovarian cancer: A retrospective study of two independent tumour tissue collections. Lancet Oncol 12: 273-285, 2011.

18. Kurashige J, Mima K, Sawada G, Takahashi Y, Eguchi H, Sugimachi K, Mori M, Yanagihara K, Yashiro M, Hirakawa K, et al: Epigenetic modulation and repression of miR-200b by cancer-associated fibroblasts contribute to cancer invasion and peritoneal dissemination in gastric cancer. Carcinogenesis 36: 133-141, 2015.

19. Shinozaki A, Sakatani T, Ushiku T, Hino R, Isogai M, Ishikawa S, Uozaki H, Takada K and Fukayama M: Downregulation of microRNA-200 in EBV-associated gastric carcinoma. Cancer Res 70: 4719-4727, 2010.

20. Song F, Yang D, Liu B, Guo Y, Zheng H, Li L, Wang T, Yu J, Zhao Y, Niu R, et al: Integrated microRNA network analyses identify a poor-prognosis subtype of gastric cancer characterized by the miR-200 family. Clin Cancer Res 20: 878-889, 2014.

21. Zhou X, Wang Y, Shan B, Han J, Zhu H, Lv Y, Fan X, Sang M, Liu XD and Liu W: The downregulation of miR-200c/141 promotes ZEB1/2 expression and gastric cancer progression. Med Oncol 32: 428, 2015.

22. Akagi T and Kimoto T: Human cell line (HGC-27) derived from the metastatic lymph node of gastric cancer. Acta Med Okayama 30: 215-219, 1976.

23. Akiyama S, Amo H, Watanabe T, Matsuyama M, Sakamoto J, Imaizumi M, Ichihashi $\mathrm{H}$, Kondo T and Takagi $\mathrm{H}$ : Characteristics of three human gastric cancer cell lines, NU-GC-2, NU-GC-3 and NU-GC-4. Jpn J Surg 18: 438-446, 1988.

24. Kakinuma N, Sato M, Yamada T, Kohu K, Nakajima M, Akiyama T, Ohwada S and Shibanaka Y: Cloning of novel LERGU mRNAs in GPR30 3' untranslated region and detection of 2 bp-deletion polymorphism in gastric cancer. Cancer Sci 96: 191-196, 2005.

25. Motoyama T, Hojo $\mathrm{H}$ and Watanabe $\mathrm{H}$ : Comparison of seven cell lines derived from human gastric carcinomas. Acta Pathol Jpn 36: $65-83,1986$.

26. Konno-Shimizu M, Yamamichi N, Inada K, Kageyama-Yahara N, Shiogama K, Takahashi Y, Asada-Hirayama I, YamamichiNishina M, Nakayama C, Ono S, et al: Cathepsin E is a marker of gastric differentiation and signet-ring cell carcinoma of stomach: A novel suggestion on gastric tumorigenesis. PLoS One 8: e56766, 2013.

27. Yamada Y, Yoshida T, Hayashi K, Sekiya T, Yokota J, Hirohashi S, Nakatani K, Nakano H, Sugimura T and Terada M: p53 gene mutations in gastric cancer metastases and in gastric cancer cell lines derived from metastases. Cancer Res 51: 5800$5805,1991$.

28. Tanaka M, Oikawa K, Takanashi M, Kudo M, Ohyashiki J, Ohyashiki K and Kuroda M: Down-regulation of miR-92 in human plasma is a novel marker for acute leukemia patients. PLoS One 4: e5532, 2009. 
29. Wu W, Takanashi M, Borjigin N, Ohno SI, Fujita K, Hoshino S, Osaka Y, Tsuchida A and Kuroda M: MicroRNA-18a modulates STAT3 activity through negative regulation of PIAS3 during gastric adenocarcinogenesis. Br J Cancer 108: 653-661, 2013.

30. Acs G, Zhang PJ, McGrath CM, Acs P, McBroom J, Mohyeldin A, Liu S, Lu H and Verma A: Hypoxia-inducible erythropoietin signaling in squamous dysplasia and squamous cell carcinoma of the uterine cervix and its potential role in cervical carcinogenesis and tumor progression. Am J Pathol 162: 1789-1806, 2003.

31. Chang L, Guo F, Huo B, Lv Y, Wang Y and Liu W: Expression and clinical significance of the microRNA-200 family in gastric cancer. Oncol Lett 9: 2317-2324, 2015.

32. Tamura G, Sakata K, Nishizuka S, Maesawa C, Suzuki Y, Iwaya T, Terashima M, Saito K and Satodate R: Inactivation of the E-cadherin gene in primary gastric carcinomas and gastric carcinoma cell lines. Jpn J Cancer Res 87: 1153-1159, 1996.

33. Murai T, Yamada S, Fuchs BC, Fujii T, Nakayama G, Sugimoto H, Koike M, Fujiwara M, Tanabe KK and Kodera Y: Epithelial-tomesenchymal transition predicts prognosis in clinical gastric cancer. J Surg Oncol 109: 684-689, 2014.

34. Elson-Schwab I, Lorentzen A and Marshall CJ: MicroRNA-200 family members differentially regulate morphological plasticity and mode of melanoma cell invasion. PLoS One 5: e13176, 2010.
35. Doki Y, Shiozaki H, Tahara H, Inoue M, Oka H, Iihara K, Kadowaki T, Takeichi $M$ and Mori T: Correlation between E-cadherin expression and invasiveness in vitro in a human esophageal cancer cell line. Cancer Res 53: 3421-3426, 1993.

36. Ji M, Lee EJ, Kim KB, Kim Y, Sung R, Lee SJ, Kim DS and Park SM: HDAC inhibitors induce epithelial-mesenchymal transition in colon carcinoma cells. Oncol Rep 33: 2299-2308, 2015.

37. Takeichi M: The cadherins: Cell-cell adhesion molecules controlling animal morphogenesis. Development 102: 639-655, 1988.

38. Castilla MÁ, Moreno-Bueno G, Romero-Pérez L, Van De Vijver K, Biscuola M, López-García MÁ, Prat J, Matías-Guiu X, Cano A, Oliva E, et al: Micro-RNA signature of the epithelialmesenchymal transition in endometrial carcinosarcoma. J Pathol 223: 72-80, 2011

39. Shao Y, Geng Y, Gu W, Huang J, Pei H and Jiang J: Prognostic role of tissue and circulating microRNA-200c in malignant tumors: A systematic review and meta-analysis. Cell Physiol Biochem 35: 1188-1200, 2015.

This work is licensed under a Creative Commons Attribution-NonCommercial-NoDerivatives 4.0 International (CC BY-NC-ND 4.0) License. 